\title{
More about the Mass of the New Charmonium States
}

\author{
T. M. Aliev, ${ }^{1,2}$ H. Özşahin, ${ }^{1}$ and M. Savcı' ${ }^{1}$ \\ ${ }^{1}$ Physics Department, Middle East Technical University, 06800 Ankara, Turkey \\ ${ }^{2}$ Institute of Physics, Baku, Azerbaijan \\ Correspondence should be addressed to M. Savc1; savci@metu.edu.tr
}

Received 13 November 2014; Revised 20 January 2015; Accepted 20 January 2015

Academic Editor: Chao-Qiang Geng

Copyright (C) 2015 T. M. Aliev et al. This is an open access article distributed under the Creative Commons Attribution License, which permits unrestricted use, distribution, and reproduction in any medium, provided the original work is properly cited. The publication of this article was funded by $\mathrm{SCOAP}^{3}$.

If $X(3872)$ is described by the picture as a mixture of the charmonium and molecular $D^{*} D$ states; $Y(3940)$ as a mixture of the $\chi_{c 0}$ and $D^{*} D^{*}$ states; and $Y(4260)$ as a mixture of the tetraquark and charmonium states, their orthogonal combinations should also exist. We estimate the mass and residues of the states within the QCD sum rules method. We find that the mass splitting among $X, Y$ and their orthogonal states is at most $200 \mathrm{MeV}$. Experimental search of these new states can play critical role for establishing the nature of the new charmonium states.

\section{Introduction}

Last decade was quite an exciting and productive period in particle physics. Starting from the first observation of the $X(3872)$ meson by the Belle Collaboration [1] up to now more than twenty new charmonium states have been observed (for the experimental and theoretical reviews and the references to the original works on the charmonium spectroscopy, see, e.g., [2-8]). The main lesson from these discoveries is that these states (referred to as $X Y Z$ states) can not be described by the simple quark model, that is, as a quark-antiquark system, and they are believed to have more complex structures. Understanding the structure and dynamics of these states is one of the hot problems in particle physics. In investigating the properties of these states two pictures are widely used, namely, four-quark (tetraquark) or meson molecules (bound states of two mesons).

The mass and some of the strong coupling constants of the $X Y Z$ mesons with light mesons are widely studied within the QCD sum rules method [9] in many works (see, e.g., $[10,11])$. The first calculation of the mass of the $X(3872)$ meson as a tetraquark state with the quantum numbers $J^{P C}=1^{++}$is performed within QCD sum rules method in
[10]. The $X(3872)$ meson as a $D^{*} D$ molecular state with the interpolating current,

$$
j_{\mu}^{\mathrm{mol}}=\frac{1}{\sqrt{2}}\left[\left(\bar{q}_{a} \gamma_{5} c_{a}\right)\left(\bar{c}_{b} \gamma_{\mu} q_{b}\right)-\left(\bar{q}_{a} \gamma_{\mu} c_{a}\right)\left(\bar{c}_{b} \gamma_{5} q_{b}\right)\right],
$$

was investigated in [11]. The next step in analysis of the properties of $X(3872)$ is that it is assumed to be a mixture of the charmonium and $D^{*} D$ molecules $[12,13]$, whose interpolating current has the form

$$
j_{\mu}^{(1)}=\cos \theta_{1} j_{\mu}^{\mathrm{ch}}+\sin \theta_{1} j_{\mu}^{\mathrm{mol}}
$$

where

$$
j_{\mu}^{\text {ch }}=\frac{1}{6 \sqrt{2}}\langle\bar{q} q\rangle\left(\bar{c} \gamma_{\mu} \gamma_{5} c\right)
$$

and $j_{\mu}^{\mathrm{mol}}$ is given in (1). In [12, 13], the mixing angle is calculated to have the value $\theta_{1}=(9 \pm 4)^{0}$ in order to reproduce the experimental value of the mass of the $X(3872)$ meson.

As far as the the state $Y(3940)$ is concerned, it is described by the mixture of the scalar $\chi_{c 0}$ and $D^{*} D^{*}$ molecules [14]. In other words its interpolating current can be written as

$$
j^{(2)}=\cos \theta_{2}\left(\frac{-\langle\bar{q} q\rangle}{\sqrt{2}} \bar{c} c\right)+\sin \theta_{2}\left(\bar{q} \gamma_{\mu} c\right)\left(\bar{c} \gamma^{\mu} q\right) .
$$


In the same manner, the mixing angle is calculated by requiring that it should reproduce the mass of the $Y(3940)$ state and is found to have the value $\theta_{2}=(76 \pm 5)^{0}$.

Finally, if one assumes that $Y(4260)$ state is a mixture of the tetraquark and the charmonium current given as [15],

$$
j_{\mu}^{(3)}=\cos \theta_{3} j_{\mu}^{\text {ch }}+\sin \theta_{3} j_{\mu}^{\text {tet }},
$$

where

$$
\begin{gathered}
j_{\mu}^{\prime \text { ch }}=\frac{1}{\sqrt{2}}\langle\bar{q} q\rangle\left(\bar{c} \gamma_{\mu} c\right), \\
j_{\mu}^{\text {tet }}=\frac{1}{\sqrt{2}} \varepsilon^{a b c} \varepsilon^{d e c} \\
{\left[\left(q_{a}^{T} C \gamma_{5} c_{b}\right)\left(\bar{q}_{d} \gamma_{\mu} \gamma_{5} C \bar{c}_{e}^{T}\right)\right.} \\
\left.+\left(q_{a}^{T} C \gamma_{\mu} \gamma_{5} c_{b}\right)\left(\bar{q}_{d} \gamma_{5} C \bar{c}_{e}^{T}\right)\right],
\end{gathered}
$$

and requires that it should reproduce the mass $Y(4260)$ state, the mixing angle is calculated to be $\theta_{3}=(53 \pm 5)^{0}$ [15]. It should be noted here that $X(3872), Y(3940)$, and $Y(4260)$, as well as other related states which are represented as the fourquark states within the QCD sum rules method, are studied in [16], [17], [18], and [19], respectively.

If the correct pictures of $X(3872), Y(3940)$, and $Y(4260)$ states were mixture of the molecular or tetraquark states with charmonium states, the orthogonal combinations of these states may also exist. In other words, these orthogonal $X^{\prime}, Y^{\prime}$, and $Y^{\prime \prime}$ states should be described by the following interpolating currents:

$$
\begin{gathered}
j_{\mu}^{(1) \prime}=-\sin \theta_{1} j_{\mu}^{\mathrm{ch}}+\cos \theta_{1} j_{\mu}^{\mathrm{mol}}, \\
j^{(2) \prime}=-\sin \theta_{2}\left(\frac{-\langle\bar{q} q\rangle}{\sqrt{2}} \bar{c} c\right)+\cos \theta_{2}\left(\bar{q} \gamma_{\mu} c\right)\left(\bar{c} \gamma^{\mu} q\right), \\
j_{\mu}^{(3) \prime}=-\sin \theta_{3} j_{\mu}^{\mathrm{ch}}+\cos \theta_{3} j_{\mu}^{\mathrm{tet}} .
\end{gathered}
$$

By imposing the condition that the states defined by the currents in (2), (4), and (5) should be orthogonal to the states defined by the currents given in (7), (8), and (9), the corresponding mixing angles are estimated within the QCD sum rules method in [20], whose values are calculated to have the values $\theta_{1}=(2.4 \pm 0.6)^{0}, \theta_{2}=(20.0 \pm 2.0)^{0}$, and $\theta_{3}=(20.0 \pm 3.0)^{0}$, respectively.

The aim of this paper is to calculate the mass and residues of these states described by the interpolating currents (7), (8), and (9).

Consider the following two-point correlation functions:

$$
\begin{aligned}
\Pi_{\mu \nu}^{(1)} & =i \int d^{4} x e^{i q x}\left\langle 0\left|T\left\{j_{\mu}^{(1) \prime}(x) \bar{j}_{\nu}^{(1) \prime}(0)\right\}\right| 0\right\rangle \\
& =\Pi^{(1)}\left(q^{2}\right)\left(g_{\mu \nu}-\frac{q_{\mu} q_{\nu}}{q^{2}}\right)+\Pi_{0}^{(1)} \frac{q_{\mu} q_{\nu}}{q^{2}}
\end{aligned}
$$

$$
\begin{aligned}
\Pi^{(2)} & =i \int d^{4} x e^{i q x}\left\langle 0\left|T\left\{j^{(2) \prime}(x) \bar{j}^{(2) \prime}(0)\right\}\right| 0\right\rangle \\
& =\Pi^{(2)}\left(q^{2}\right), \\
\Pi_{\mu \nu}^{(3)} & =i \int d^{4} x e^{i q x}\left\langle 0\left|T\left\{j_{\mu}^{(3) \prime}(x) \bar{j}_{\nu}^{(3) \prime}(0)\right\}\right| 0\right\rangle \\
& =\Pi^{(3)}\left(q^{2}\right)\left(g_{\mu \nu}-\frac{q_{\mu} q_{\nu}}{q^{2}}\right)+\Pi_{0}^{(3)} \frac{q_{\mu} q_{\nu}}{q^{2}} .
\end{aligned}
$$

According to the duality principle the phenomenological part of these correlation functions can be calculated in terms of quarks, gluons, and hadrons by inserting complete set of hadrons carrying the same quantum numbers as the interpolating currents themselves. Then isolating the ground states and performing summation over the spins (if ever exist) we get

$$
\begin{gathered}
\Pi_{\mu \nu}^{(1)}=\frac{\left\langle 0\left|j_{\mu}^{(1)}\right| B(q)\right\rangle\left\langle B(q)\left|j_{\nu}^{(1)}\right| 0\right\rangle}{q^{2}-m_{B_{1}}^{2}}+\cdots \\
=\frac{\lambda^{(1) 2}}{m_{B_{1}}^{2}-q^{2}}\left(-g_{\mu \nu}+\frac{q_{\mu} q_{\nu}}{m_{B_{1}}^{2}}\right)+\cdots \\
\Pi^{(2)}=\frac{\lambda^{(2) 2}}{m_{B_{2}}^{2}-q^{2}}+\cdots \\
\Pi_{\mu \nu}^{(3)}=\frac{\lambda^{(3) 2}}{m_{B_{3}}^{2}-q^{2}}\left(-g_{\mu \nu}+\frac{q_{\mu} q_{\nu}}{m_{B_{1}}^{2}}\right)+\cdots,
\end{gathered}
$$

where $\cdots$ means the contributions of the higher states and continuum, and we have used

$$
\begin{gathered}
\left\langle 0\left|j_{\mu}^{(1),(3)}\right| B(q)\right\rangle=\lambda^{(1),(3)} \varepsilon_{\mu}^{(1),(3)}, \\
\left\langle 0\left|j^{(2)}\right| B(q)\right\rangle=\lambda^{(2)} .
\end{gathered}
$$

The continuum contribution to the spectral density is modeled as a spectral density from the operator product expansion (OPE) starting from some threshold $s_{0}$; that is,

$$
\rho^{\mathrm{cont}}(s)=\rho^{\mathrm{OPE}} \theta\left(s-s_{0}\right),
$$

where $\theta\left(s-s_{0}\right)$ is the Heaviside step function.

In order to construct the sum rules for the mass and residues of new charmonium states the calculation of the correlation functions in terms of the quark and gluon degrees of freedom using the operator product expansion (OPE) are needed. On the other hand, to be able to calculate the correlation functions from QCD side the heavy and light quark propagators are needed. The expression for the 
propagator of the light quark in the coordinate space is given as

$$
\begin{aligned}
S_{q}(x)= & \frac{i \not x}{2 \pi^{2} x^{4}}-\frac{m_{q}}{4 \pi^{2} x^{2}} \\
& -\frac{\langle\bar{q} q\rangle}{12}\left(1-\frac{i m_{q}}{4} \not x\right)-\frac{x^{2}}{192} m_{0}^{2}\langle\bar{q} q\rangle\left(1-\frac{i m_{q}}{6} \not x\right) \\
& -\frac{\alpha_{s} \pi x^{2} \not x}{2^{3} 3^{5}}\langle\bar{q} q\rangle^{2}+\frac{1}{32 \pi^{2}} g_{s} G_{\mu \nu} \frac{\sigma^{\mu \nu} \not x+\not x \sigma^{\mu \nu}}{x^{2}} \\
& -\frac{\pi^{2} x^{4}}{2^{8} 3^{3}}\langle\bar{q} q\rangle\left\langle g_{s}^{2} G^{2}\right\rangle \\
& -\frac{\alpha_{s} m_{q} \pi x^{4}}{2^{5} 3^{5}}\langle\bar{q} q\rangle^{2}-\frac{m_{q} x^{2} \not x}{2^{7} 3^{2}} m_{0}^{2}\langle\bar{q} q\rangle,
\end{aligned}
$$

and that of the heavy quark is given as

$$
\begin{gathered}
S_{\mathrm{Q}}=\frac{m_{\mathrm{Q}}^{2}}{4 \pi^{2}}\left[\frac{i \not x}{\left(-x^{2}\right)} K_{2}\left(m_{\mathrm{Q}} \sqrt{-x^{2}}\right)+\frac{1}{\left(\sqrt{-x^{2}}\right)} K_{1}\left(m_{\mathrm{Q}} \sqrt{-x^{2}}\right)\right] \\
-\frac{m_{\mathrm{Q}} g_{s} G_{\mu \nu}}{32 \pi^{2}}\left[\frac{i\left(\sigma^{\mu \nu} \not x+\not x \sigma^{\mu \nu}\right)}{\left(\sqrt{-x^{2}}\right)} K_{1}\left(m_{\mathrm{Q}} \sqrt{-x^{2}}\right)\right. \\
\left.+2 \sigma^{\mu \nu} K_{0}\left(m_{\mathrm{Q}} \sqrt{-x^{2}}\right)\right] \\
-\frac{\left\langle g_{s}^{2} G^{2}\right\rangle}{2304 \pi^{2}}\left[\left(i m_{\mathrm{Q}} \not x-6\right)\left(\sqrt{-x^{2}}\right) K_{1}\left(m_{\mathrm{Q}} \sqrt{-x^{2}}\right)\right. \\
\left.+m_{\mathrm{Q}}\left(-x^{2}\right) K_{2}\left(m_{\mathrm{Q}} \sqrt{-x^{2}}\right)\right]
\end{gathered}
$$

(see [21] and [22], resp.).

The theoretical part of the correlation function(s) can be written in terms of the dispersion relation as

$$
\Pi^{(i)}\left(q^{2}\right)=\int_{4 m_{c}^{2}}^{\infty} d s \frac{\rho^{\mathrm{OPE}^{(i)}}(s)}{s-q^{2}} .
$$

Choosing the coefficient of the $g_{\mu \nu}$ structure for the correlators (10) and (12), for the spectral densities we get

$$
\begin{aligned}
\rho^{(1)}(s)= & \frac{1}{4096 \pi^{6}} \\
& \cdot \int_{\alpha_{\min }}^{\alpha_{\max }} d \alpha \int_{\beta_{\min }}^{\beta_{\max }} d \beta \\
& \cdot\left\{3 \alpha \beta\left[1-(\alpha+\beta)^{2}\right] \mu_{1}^{4}\right. \\
& \quad-6 m_{c} m_{q}(\alpha+\beta)(1-\alpha-\beta)(3-\alpha+\beta) \mu_{1}^{3} \\
& -48 \pi^{2}\left[(\alpha+\beta)(1+\alpha+\beta) m_{c}+2 \alpha \beta m_{q}\right] \\
& \cdot\langle\bar{q} q\rangle \mu_{1}^{2}
\end{aligned}
$$

$$
\left.+24 \pi^{2}\left[16 m_{c} m_{q}+(\alpha+\beta) m_{0}^{2}\right] m_{c}\langle\bar{q} q\rangle \mu_{1}\right\}
$$$$
\cdot \cos ^{2} \theta+\frac{1}{1536 \pi^{4}}
$$$$
\cdot\left\{36 \alpha(1-\alpha) m_{q}\langle\bar{q} q\rangle \mu_{2}^{2} \cos ^{2} \theta\right.
$$$$
-2\left(m_{0}^{2}\left[9 m_{c}-6 \alpha(1-\alpha) m_{q}\right]\right.
$$$$
\left.+8 \pi^{2} \alpha(1-\alpha)\langle\bar{q} q\rangle\right)
$$

$\cdot\langle\bar{q} q\rangle \mu_{2} \cos ^{2} \theta$

$$
\begin{aligned}
+4 & \left(3 m_{0}^{2} m_{q}\left[3 m_{c}^{2}-\alpha(1-\alpha) s\right]\right. \\
& \left.+2 \pi^{2}\left[10 m_{c}^{2}-9 m_{c} m_{q}+2 \alpha(1-\alpha) s\right]\langle\bar{q} q\rangle\right)
\end{aligned}
$$

$\cdot\langle\bar{q} q\rangle \cos ^{2} \theta$

$$
\begin{aligned}
& -16 \pi^{2}\left[m_{c}^{2}+\alpha(1-\alpha)\left(\mu_{2}-s\right)\right] \\
& \left.\cdot\langle\bar{q} q\rangle^{2} \sin \theta(\sin \theta+2 \cos \theta)\right\} \\
\rho^{(2)}(s)=\frac{1}{512 \pi^{6}} & \int_{\alpha_{\min }}^{\alpha_{\max }} d \alpha \int_{\beta_{\min }}^{\beta_{\max }} d \beta \\
\cdot\{ & -3 \alpha \beta(1-\alpha-\beta) \mu_{1}^{4} \\
& +6(1-\alpha-\beta)(\alpha+\beta) m_{c} m_{q} \mu_{1}^{3} \\
& +24 \pi^{2}(\alpha+\beta) m_{c}\langle\bar{q} q\rangle \mu_{1}^{2} \\
& \left.-192 \pi^{2} m_{c}^{2} m_{q}\langle\bar{q} q\rangle \mu_{1}\right\} \cos ^{2} \theta+\frac{1}{384 \pi^{4}} \\
& +48 \pi^{2}\left[m_{c}^{2}+\alpha(1-\alpha)\left(2 \mu_{2}-s\right)\right] \\
& \left.\cdot\left\langle\int_{\alpha_{\min }}^{\alpha_{\max }} d \alpha\right\rangle^{2} \sin \theta(3 \sin \theta-2 \sqrt{2} \cos \theta)\right\} \\
\cdot\{ & -36 \alpha(1-\alpha) m_{q}\langle\bar{q} q\rangle \mu_{2}^{2} \cos ^{2} \theta \\
& +\left(3 m_{0}^{2}\left[3 m_{c}-8 \alpha(1-\alpha) m_{q}\right]\right. \\
& +\langle\bar{q} q\rangle \mu_{2} \cos ^{2} \theta \\
& \left.+64 \pi^{2} \alpha(1-\alpha)\langle\bar{q} q\rangle\right) \\
& \left.\cdot\left[m_{c}\left(3 m_{q}-4 m_{c}^{2}\right)-2 \alpha(1-\alpha) s\right]\langle\bar{q} q\rangle\right) \\
& \\
& \\
& \\
&
\end{aligned}
$$




$$
\begin{aligned}
\rho^{(3)}(s)= & \frac{1}{3072 \pi^{6}} \\
& \cdot \int_{\alpha_{\min }}^{\alpha_{\max }} d \alpha \int_{\beta_{\min }}^{\beta_{\max }} d \beta \\
& \cdot\left\{3 \alpha \beta\left[1-(\alpha+\beta)^{2}\right] \mu_{1}^{4}-2(1-\alpha-\beta)^{3} m_{c}^{2} \mu_{1}^{3}\right. \\
& +192 \pi^{2} \alpha \beta m_{q}\langle\bar{q} q\rangle \mu_{1}^{2} \\
& -96 \pi^{2}(5-\alpha-\beta) m_{c}^{2} m_{q}\langle\bar{q} q\rangle \mu_{1} \\
& \left.-16 \pi^{2} m_{0}^{2} m_{c}^{2} m_{q}\langle\bar{q} q\rangle\right\} \cos ^{2} \theta+\frac{1}{192 \pi^{4}} \\
& \cdot \int_{\alpha_{\min }}^{\alpha_{\max }} d \alpha \\
& \cdot\left\{\alpha(1-\alpha)\langle\bar{q} q\rangle \mu_{2}\right. \\
& \cdot\left[6 m_{q} \mu_{2}-\left(m_{0}^{2} m_{q}+16 \pi^{2}\langle\bar{q} q\rangle\right)\right] \cos ^{2} \theta \\
& -\left(2 m_{0}^{2} m_{q}\left[3 m_{c}^{2}+\alpha(1-\alpha) s\right]\right. \\
& \left.+8 \pi^{2}\left[m_{c}^{2}+\alpha(1-\alpha)\left(m_{0}^{2}-s\right)\right]\langle\bar{q} q\rangle\right) \\
& \cdot\langle\bar{q} q\rangle \cos ^{2} \theta \\
& +24 \pi^{2}\left[m_{c}^{2}-\alpha(1-\alpha)\left(\mu_{2}-s\right)\right] \\
& \left.\cdot\langle\bar{q} q\rangle^{2} \sin \theta(3 \sin \theta-\cos \theta)\right\} \\
&
\end{aligned}
$$

where

$$
\begin{gathered}
\mu_{1}=\frac{m_{c}^{2}(\alpha+\beta)}{\alpha \beta}-s, \\
\mu_{2}=\mu_{1}(\beta \longrightarrow 1-\alpha), \\
\beta_{\min }=\frac{\alpha m_{c}^{2}}{\alpha s-m_{c}^{2}}, \\
\beta_{\max }=1-\alpha, \\
\alpha_{\min }=\frac{1}{2}(1-v), \\
\alpha_{\max }=\frac{1}{2}(1+v), \\
v=\sqrt{1-\frac{4 m_{c}^{2}}{s}} .
\end{gathered}
$$

For simplicity, in these expressions we do not present the terms proportional with the gluon condensate $\left\langle g_{s}^{2} G^{2}\right\rangle$. It should be noted that these spectral densities are all calculated in [12-15]. Perturbative parts of our results coincide with those presented in [12-15], while there are some discrepancies in quark condensates and $d=5$ operators. These differences are due to the fact that some of the contributions corresponding to the quark condensates and $d=5$ operators are neglected in [12-15].

Equating the spectral densities given in (19), (20), and (21) with the coefficients of $\Pi^{(1)}, \Pi^{(2)}$, and $\Pi^{(3)}$, respectively, and performing Borel transformation over $-q^{2}$, we get

$$
\lambda^{(i) 2} e^{-m_{1}^{2} / M^{2}}=\int_{4 m_{c}^{2}}^{s_{0}} d s \rho^{(i)}(s) e^{-s / M^{2}},
$$

where $i=1,2,3$. In deriving (23) the quark-hadron duality ansatz has been used; that is, the contribution of the continuum and higher states are assumed to be the same as the perturbative ones starting on from the threshold.

In order to obtain the mass sum rules for the mass, we take the derivative of (23) with respect to $1 / M^{2}$ on both sides and divide the obtained result by itself, from which we obtain

$$
m_{i}^{2}=\frac{\int_{4 m_{c}^{2}}^{s_{0}} d s s \rho^{(i)}(s) e^{-s / M^{2}}}{\int_{4 m_{c}^{2}}^{s_{0}} d s \rho^{(i)} e^{-s / M^{2}}} .
$$

\section{Numerical Analysis}

Here in this section we present the results of the numerical analysis on the mass and residues of the considered new charmonium states. For the $c$-quark masses we have used its $\overline{M S}$ scheme masses, $\bar{m}_{c}\left(\bar{m}_{c}\right)=(1.28 \pm 0.03) \mathrm{GeV}$ [23], $\langle\bar{q} q\rangle=$ $(1 \mathrm{GeV})=-\left(0.246_{-19}^{+28} \mathrm{MeV}\right)^{3}[24], m_{0}^{2}=(0.8 \pm 0,2) \mathrm{GeV}^{2}$, $\langle\bar{s} s\rangle=0.8\langle\bar{q} q\rangle$ [25], and $m_{s}(2 \mathrm{GeV})=(102 \pm 8) \mathrm{MeV}$ [26].

The sum rules for the mass and residue contain two auxiliary variables, namely, the Borel mass parameter $M^{2}$ and the continuum threshold $s_{0}$. Any physical quantity should be independent of them, and therefore our primary aim is to find the regions of $M^{2}$ and $s_{0}$, where the mass and residue are practically independent of them. It should be noted here that the continuum threshold itself is not a totally arbitrary parameter but related to the first excited state. In general, the quantity $\sqrt{s_{0}}-m$, where $m$ is the mass of the ground state, corresponds to the energy of the first excited state with the same quantum number. The range of this quantity typically changes in the region 0.3 to $0.8 \mathrm{GeV}$. The lower limit of $M^{2}$ is determined by requiring that the operator product expansion series is convergent. In other words the contribution of the perturbative part must dominate over the nonperturbative one. The upper bound of $M^{2}$ is decided from the condition that the contribution coming from the continuum constitutes about $1 / 3$ of the contribution coming from the perturbative part, that is, the ratio

$$
R=\frac{\int_{s_{0}}^{\infty} d s \rho(s) e^{-s / M^{2}}}{\int_{4 m_{c}^{2}}^{\infty} d s \rho(s) e^{-s / M^{2}}}<\frac{1}{3},
$$


which leads to the following "working regions" of the Borel mass parameter:

$$
\begin{array}{ll}
2.4 \leq M^{2} \leq 4.0 \mathrm{GeV}^{2} & \text { for } X^{\prime} \text { state, } \\
2.5 \leq M^{2} \leq 4.0 \mathrm{GeV}^{2} & \text { for } Y^{\prime} \text { state, } \\
2.5 \leq M^{2} \leq 5.0 \mathrm{GeV}^{2} & \text { for } Y^{\prime \prime} \text { state }
\end{array}
$$

In Figure 1, we present the dependence of the mass of $X^{\prime}$ state on $M^{2}$, at the fixed value of the continuum threshold $\sqrt{s_{0}}=4.4 \mathrm{GeV}$ and of the mixing angle $\theta$. We observe from these figures that, in the considered domains of $M^{2}$, the results exhibit good stability with respect to the variation in $M^{2}$ and seem to be practically insensitive to the variations in $s_{0}$ and the mixing angle $\theta$. As has already been noted, if $X$ meson is represented as mixture of the charmonium and $D^{*} D$ molecular states, it is found in [20] the mixing angle is equal to $\theta=(2.4 \pm 0.3)^{0}$. In other words, in this picture $X(3872)$ state can be said to be composed of pure charmonium and its orthogonal combination $X^{\prime}$ which is described by the $D^{*} D$ molecular state. The study of the decay channels of $X(3872)$ and $X^{\prime}$ states can give unambiguous useful information about the "correct" pictures of these states. Our final result for the mass of $X^{\prime}$ is that

$$
m_{X^{\prime}}=(3.75 \pm 0.15) \mathrm{GeV}
$$

In Figure 2 we present the $M^{2}$ dependence of the residue of $X^{\prime}$ on $M^{2}$ for the mixing angle $\theta=2.4^{0}$, at the fixed value of the continuum threshold $\sqrt{s_{0}}=4.4 \mathrm{GeV}$. We observe that the residue is weakly dependent on $M^{2}$, and we deduce from this figure that

$$
\lambda_{X^{\prime}}=(1.3 \times \pm 0.2) \times 10^{-2} \mathrm{GeV}^{3} .
$$

The dependencies of the mass and residue $Y^{\prime}$ state on $M^{2}$ for the mixing angle $\theta=20^{\circ}$, at $\sqrt{s_{0}}=4.4 \mathrm{GeV}$, are presented in Figures 3 and 4, respectively. We observe from these figures that, again, the mass and residue of $Y^{\prime}$ state seem to be practically insensitive with respect to the variation in $M^{2}$, whose values are calculated to be

$$
\begin{aligned}
& m_{Y^{\prime}}=(3.85 \pm 0.20) \mathrm{GeV}, \\
& \lambda_{Y^{\prime}}=(1.9 \pm 0.4) \times 10^{-2} \mathrm{GeV}^{-3} .
\end{aligned}
$$

Performing similar approach for the $Y^{\prime \prime}$ state, we see from Figures 5 and 6 that

$$
\begin{aligned}
& m_{Y^{\prime \prime}}=(4.4 \pm 0.1) \mathrm{GeV}, \\
& \lambda_{Y^{\prime \prime}}=(2.0 \pm 0.2) \times 10^{-2} \mathrm{GeV}^{-3} .
\end{aligned}
$$

We would like to note here that only $Y^{\prime \prime}$ state is sensitive to the change in the value of the mixing angle $\theta$. With the increasing value of $\theta$, the mass of the $Y^{\prime \prime}$ state is decreasing; for example, at $\theta=40^{\circ}, m_{Y^{\prime \prime}}=(4.0 \pm 0.1) \mathrm{GeV}$ and at $\theta=$ $50^{0}, m_{Y^{\prime \prime}}=(3.9 \pm 0.1) \mathrm{GeV}$.

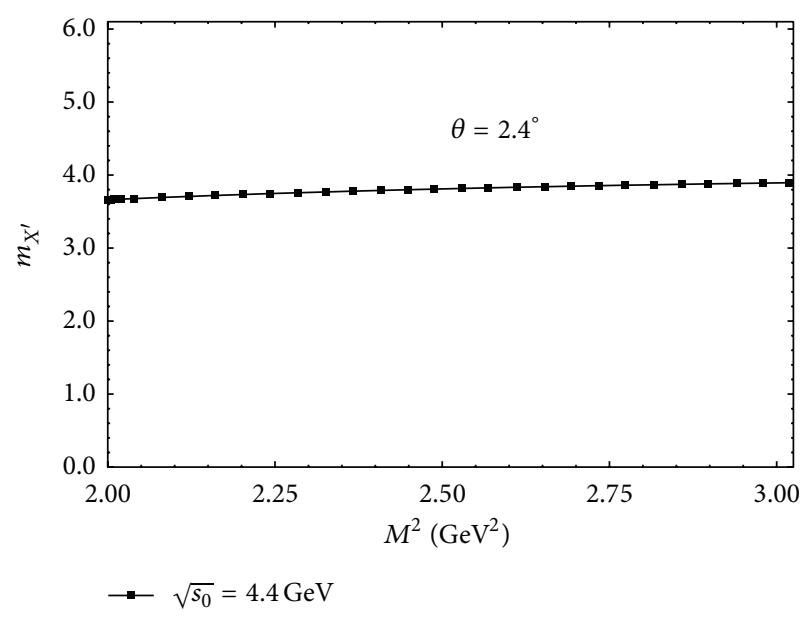

FIgURE 1: The dependence of the mass of the $X^{\prime}$ state on Borel mass square $M^{2}$ for the mixing angle $\theta=2.4^{0}$, at the fixed value of the continuum threshold $\sqrt{s_{0}}=4.4 \mathrm{GeV}$.

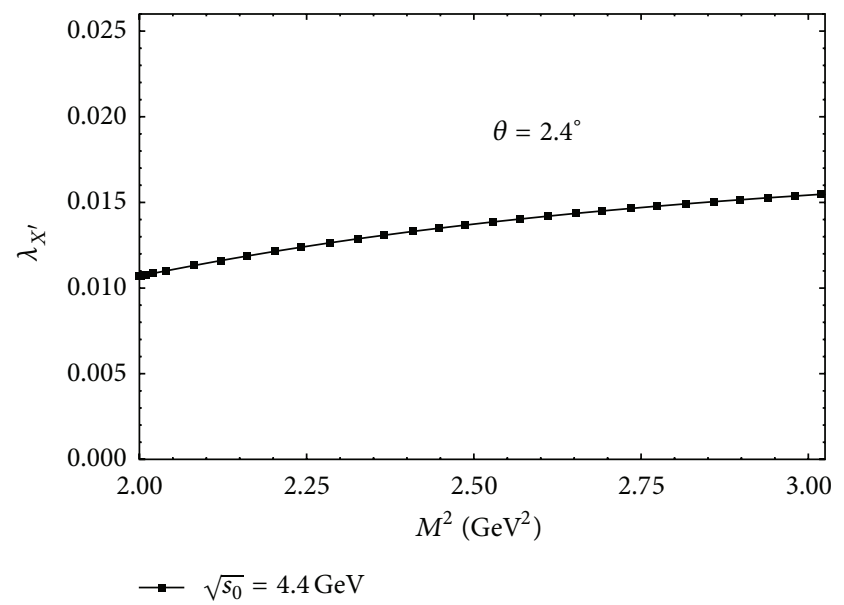

FIgURE 2: The dependence of the residue of the $X^{\prime}$ state on Borel mass square $M^{2}$ for the mixing angle $\theta=2.4^{0}$, at the fixed value of the continuum threshold $\sqrt{s_{0}}=4.4 \mathrm{GeV}$.

We also calculate the mass of the $X(3872), Y(3940)$, and $Y(4260)$ states at $\theta_{1}=(2.4 \pm 0.6)^{0}, \theta_{2}=(20 \pm 2)^{0}$, and $\theta_{3}=$ $(20 \pm 3)^{0}$, respectively, and obtain the following mass values: $m_{X(3872)} \equiv(3.65 \pm 0.10) \mathrm{GeV}, m_{Y(3940)} \equiv(3.7 \pm 0.1) \mathrm{GeV}$, and $m_{Y(4260)} \equiv(3.9 \pm 0.10) \mathrm{GeV}$. In other words, sum rules predictions for the masses are slightly lower compared to their experimental values.

From these predictions of the masses of $X^{\prime}, Y^{\prime}$, and $Y^{\prime \prime}$ states and the experimental values of $X(3872), Y(3940)$, and $Y(4260)$ states we observe that the splittings among these states are at most $100 \mathrm{MeV} \leq \Delta m \leq 200 \mathrm{MeV}$. These results can be checked in future planned experiments after the discovery of the $X^{\prime}, Y^{\prime}$, and $Y^{\prime \prime}$ states.

The predicted results for the residues of the $Y^{\prime \prime}$ and $Y(4260) ; Y^{\prime}$ and $Y(3940)$ states, respectively, are very close to each other, while the result for the $X^{\prime}$ state is three times larger compared to that of the $X(3872)$ state. 


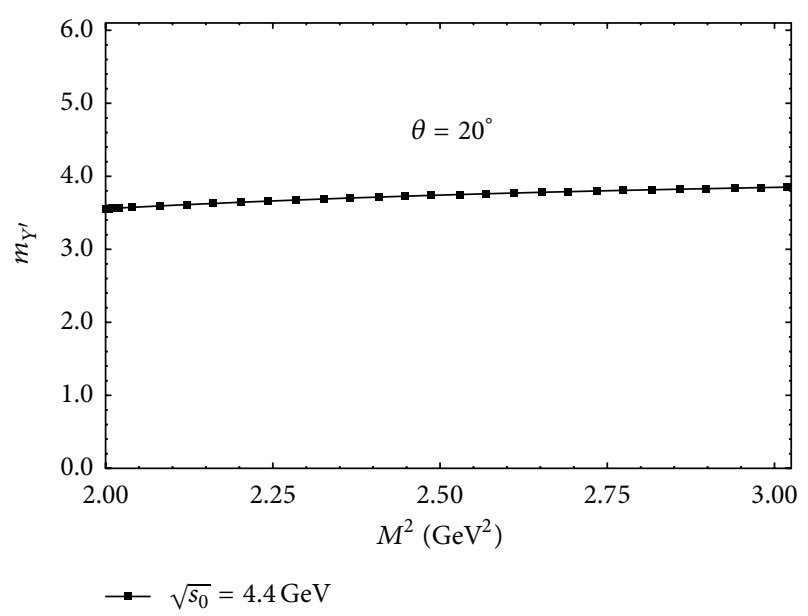

FIGURE 3: The dependence of the mass of the $Y^{\prime}$ state on Borel mass square $M^{2}$ for the mixing angle $\theta=20^{\circ}$, at the fixed value of the continuum threshold $\sqrt{s_{0}}=4.4 \mathrm{GeV}$.

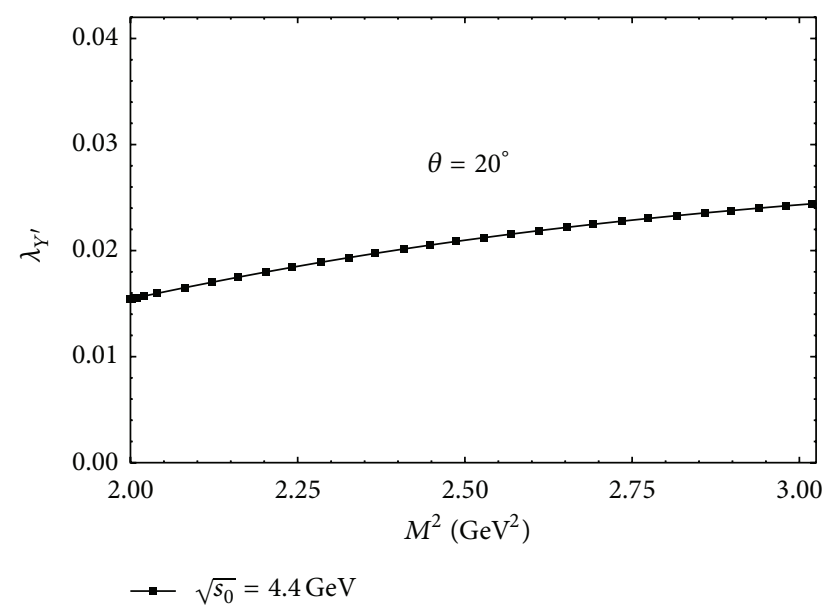

Figure 4: The dependence of the residue of the $Y^{\prime}$ state on Borel mass square $M^{2}$ for the mixing angle $\theta=20^{\circ}$, at the fixed value of the continuum threshold $\sqrt{s_{0}}=4.4 \mathrm{GeV}$.

Few words about the decay channels of these states are in order. The main decay channels of the $X(3872)$ and $X^{\prime}$ states are $\Psi \pi^{+} \pi^{-}, J / \Psi \rho, J / \Psi \omega, J / \Psi \gamma, J / \Psi \phi, \Psi^{\prime} \gamma, \chi_{c} \gamma, D \bar{D}^{*}$, and $D \bar{D} \pi$; of the $Y(4260)$ and $Y^{\prime \prime}$ states are $J / \Psi \eta, \Psi^{\prime} \pi^{+} \pi^{-}$, $D \bar{D}, D \bar{D}^{*}, \chi_{c} \gamma$, and $D D^{*} \pi$, and of the $Y(3940)$ and $Y^{\prime}$ states are $J / \Psi \omega, D \bar{D}, D^{*} \bar{D}^{*}$, and $\bar{D}^{*+} D^{-} \gamma$ (see, e.g., [2-8] and the references therein). It should especially be stressed here that the measurement of the photon spectrum in the radiative decay channels can play critical role in testing the structure of these mesons.

In conclusion, using the picture that assumes $X(3872)$ as the mixture of the charmonium and the molecular $D^{*} D$ states; $Y(3940)$ as the mixture of the $\chi_{c 0}$ and $D^{*} D^{*}$ states; and $Y(4260)$ as the mixture of the tetraquark and charmonium states, we calculate the mass and the residues of their orthogonal states within the QCD sum rules method. We obtain that the mass splittings among these states are at most

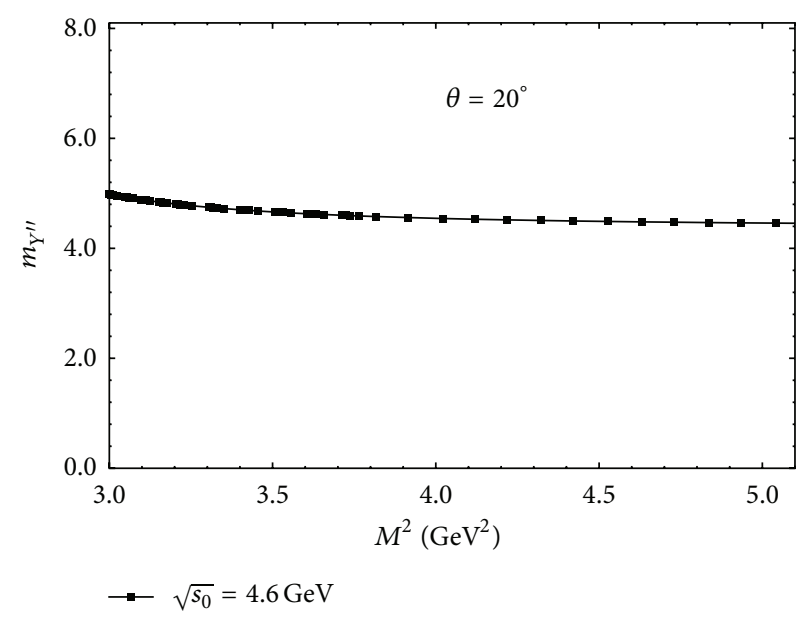

FIGURE 5: The dependence of the mass of the $Y^{\prime \prime}$ state on Borel mass square $M^{2}$ for the mixing angle $\theta=20^{\circ}$, at the fixed value of the continuum threshold $\sqrt{s_{0}}=4.6 \mathrm{GeV}$.

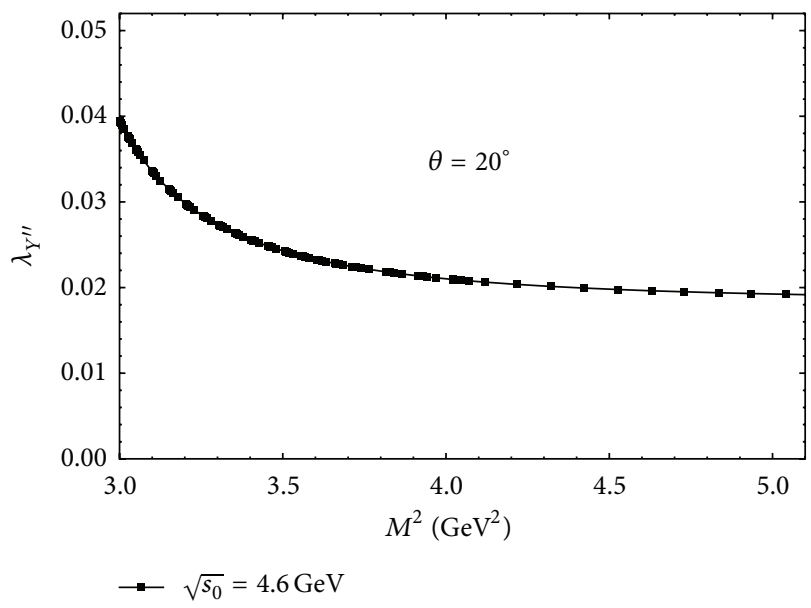

Figure 6: The dependence of the residue of the $Y^{\prime \prime}$ state on Borel mass square $M^{2}$ for the mixing angle $\theta=20^{\circ}$, at the fixed value of the continuum threshold $\sqrt{s_{0}}=4.6 \mathrm{GeV}$.

$200 \mathrm{MeV}$. Experimental search of new orthogonal states can be quite useful in establishing correct picture of these states. The study of the decay channels can also be very useful in this investigation.

\section{Conflict of Interests}

The authors declare that there is no conflict of interests regarding the publication of this paper.

\section{References}

[1] S.-K. Choi, S. L. Olsen, K. Abe et al., "Observation of a narrow charmoniumlike state in exclusive $B^{ \pm} \rightarrow K^{ \pm} \pi^{+} \pi^{-} J / \psi$ decays," Physical Review Letters, vol. 91, Article ID 262001, 2003.

[2] X. Liu, "An overview of XYZ new particles," Chinese Science Bulletin, vol. 59, no. 29-30, pp. 3815-3830, 2014. 
[3] E. S. Swanson, "The new heavy mesons: a status report," Physics Reports, vol. 429, no. 5, pp. 243-305, 2006.

[4] E. Klempt and A. Zaitsev, "Glueballs, hybrids, multiquarks: experimental facts versus QCD inspired concepts," Physics Reports, vol. 454, pp. 1-202, 2007.

[5] M. Nielsen, F. S. Navarra, and S. H. Lee, "New charmonium states in QCD sum rules: a concise review," Physics Reports, vol. 497, no. 2-3, pp. 41-83, 2010.

[6] M. Nielsen and F. S. Navarra, "Charged exotic charmonium states," Modern Physics Letters A, vol. 29, no. 5, Article ID 1430005, 2014.

[7] N. Brambilla, S. Eidelman, B. K. Heltsley et al., "Heavy quarkonium: progress, puzzles, and opportunities," The European Physical Journal C, vol. 71, article 1534, 2011.

[8] A. Esposito, A. L. Guerrieri, P. Piccinini, A. Pilloni, and A. D. Poloso, "Four-quark hadrons: an updated review," International Journal of Modern Physics A, 2014.

[9] M. A. Shifman, A. I. Vainshtein, and V. I. Zakharov, "QCD and resonance physics. Theoretical foundations," Nuclear Physics, Section B, vol. 147, no. 5, pp. 385-447, 1979.

[10] R. D. Matheus, F. S. Navarra, M. Nielsen, and C. M. Zanetti, "QCD sum rules for the $\mathrm{X}(3872)$ as a mixed moleculecharmonium state," Physical Review D, vol. 80, Article ID 056002, 2009.

[11] M. Nielsen, U. Wiedner, and S. H. Lee, " $D_{S} D^{*}$ molecule as an axial meson," Journal of the Korean Physical Society, vol. 55, no. 22, pp. 424-428, 2009.

[12] R. D. Matheus, F. S. Navarra, M. Nielsen, and C. M. Zanetti, "QCD sum rules for the production of the $\mathrm{X}(3872)$ as a mixed molecule-charmonium state in $B$ meson decay," Physics Letters B, vol. 702, pp. 359-363, 2011.

[13] R. D. Matheus, F. S. Navarra, M. Nielsen, and C. M. Zanetti, "QCD sum rules for the $X(3872)$ as a mixed moleculecharmonium state," Physical Review D, vol. 80, Article ID 056002, 2009.

[14] R. M. Albuquerque, J. M. Dias, M. Nielsen, and C. M. Zanetti, "Y(3940) as a mixed charmonium-molecule state," Physical Review D, vol. 89, Article ID 076007, 2014.

[15] J. M. Dias, R. M. Albuquerque, M. Nielsen, and C. M. Zanetti, "Y (4260) as a mixed charmonium-tetraquark state," Physical Review D, vol. 86, Article ID 116012, 2012.

[16] Z. W. Wang and T. Huang, "Possible assignments of the $X(3872)$, $Z_{c}(3900)$, and $Z_{b}(10610)$ as axial-vector molecular states," The European Physical Journal C, vol. 74, article 2891, 2014.

[17] Z.-G. Wang, "Reanalysis of the TeX, TeX, TeX, TeX, and TeX as molecular states with QCD sum rules," The European Physical Journal C, vol. 74, article 2963, 2014.

[18] J.-R. Zhang and M.-Q. Huang, " $P$-wave $[c s][\overline{c s}]$ tetraquark state: $Y(4260)$ or $Y(4660)$ ?” Physical Review D, vol. 83, Article ID 036005, 2011.

[19] Z. W. Wang, "Mass spectrum of the vector-hidden charm and bottom tetraquark states," Journal of Physics G, vol. 36, no. 8, Article ID 085002, 2009.

[20] T. M. Aliev and M. Savc1, "Determination of the mixing angle between new charmonium states," http://arxiv.org/abs/ 1409.5248 .

[21] L. Wang and F. X. Lee, "MathQCDSR: a Mathematica package for QCD sum rules calculations," Computer Physics Communications, vol. 182, no. 8, pp. 1721-1731, 2011.

[22] I. I. Balitsky and V. M. Braun, "Evolution equations for QCD string operators," Nuclear Physics, Section B, vol. 311, no. 3, pp. 541-584, 1989.
[23] K. G. Chetyrkin, J. H. Kühn, A. Maier et al., "Charm and bottom quark masses: an update," Physical Review D, vol. 80, Article ID 074010, 2009.

[24] B. L. Ioffe, "QCD (Quantum chromodynamics) at low energies," Progress in Particle and Nuclear Physics, vol. 56, no. 1, pp. 232277, 2006.

[25] V. M. Belyaev and B. L. Ioffe, "Determination of baryon and baryonic masses from QCD sum rules. Strange baryons," Soviet Physics-JETP, vol. 57, p. 716, 1983.

[26] C. A. Dominguez, N. F. Nasrallah, R. Röntsch, and K. Schilcher, "Strange quark mass from finite energy QCD sum rules to five loops," Journal of High Energy Physics, vol. 2008, no. 5, article 20, 2008 . 

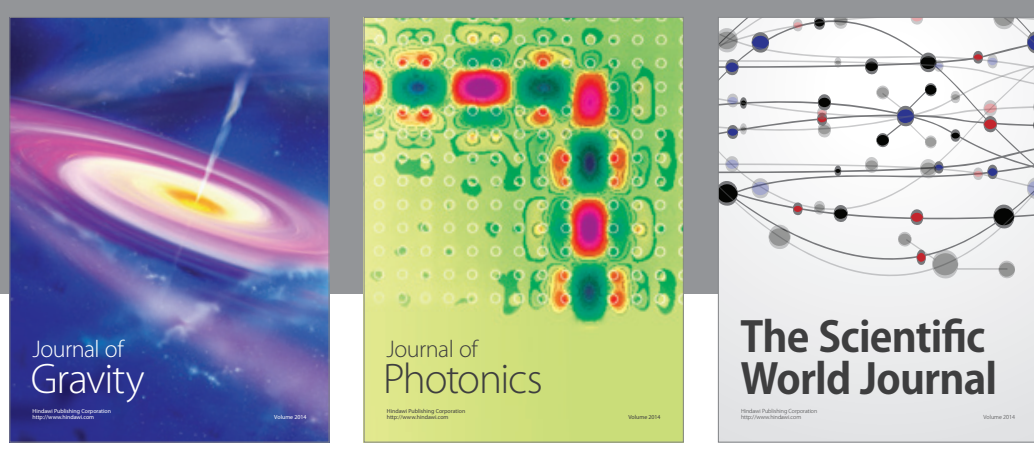

The Scientific World Journal
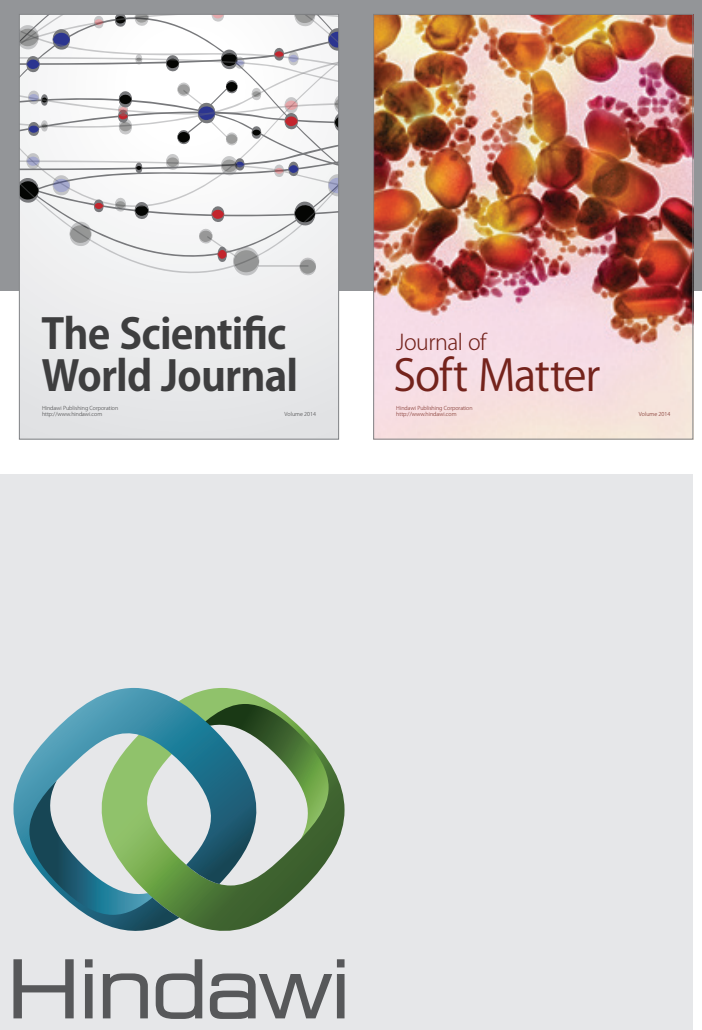

Submit your manuscripts at

http://www.hindawi.com

nternational Journal of

Statistical Mechanics
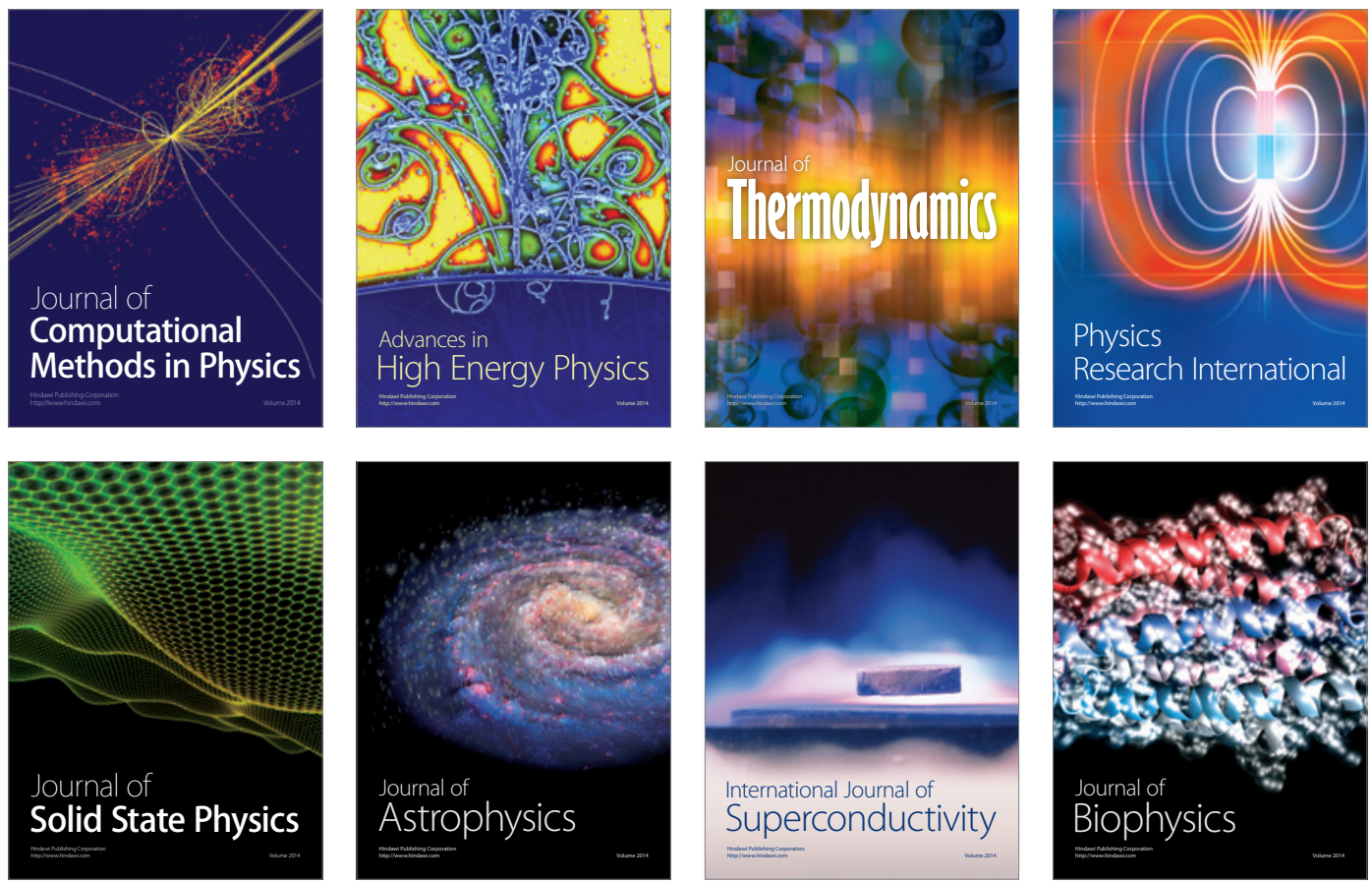
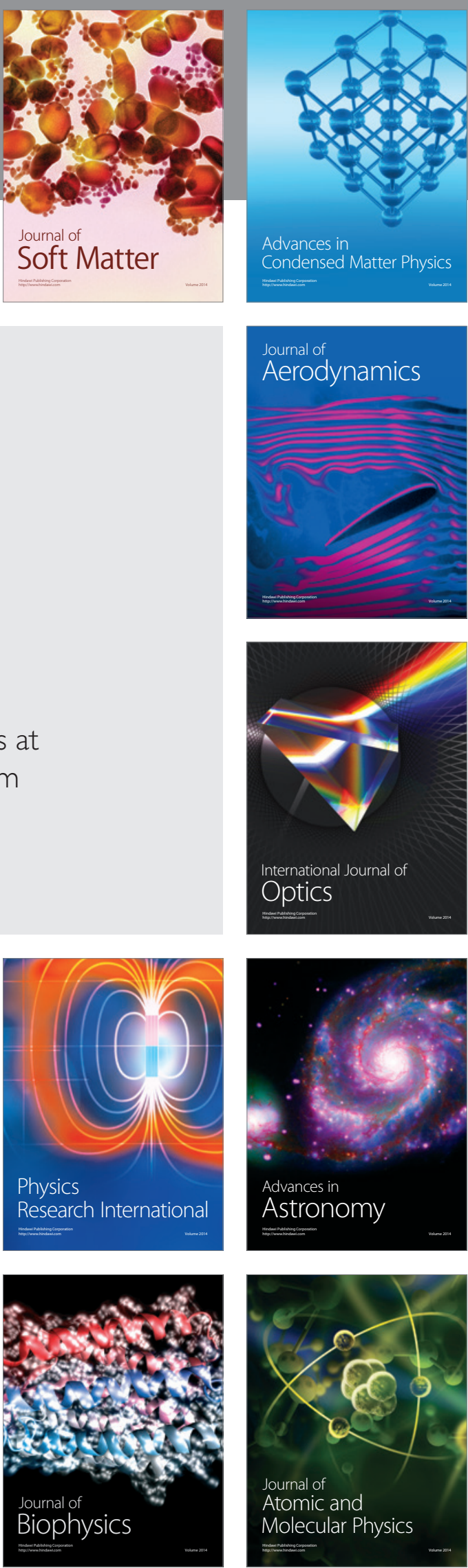